\title{
A Case of Pharyngeal-Cervical-Brachial Variant of Guillain-Barré associated with Campilobacter Jejuni Infection
}

\author{
Gabriela Pereira*, Ana Cardoso, Margarida Rocha, Ussumane Embaló, Jorge Cotter
}

Internal Medicine, Hospital Senhora da Oliveira, Guimarães, Portugal.

*Corresponding Author: Gabriela Pereira, Internal Medicine, Hospital Senhora da Oliveira, Guimarães, Portugal.

Received date: October 12, 2021; Accepted date: January 07, 2022; Published date: January 14, 2022

Citation: G Pereira, A Cardoso, M Rocha, U Embaló, J Cotter. (2022). A Case of Pharyngeal-Cervical-Brachial Variant of Guillain-Barré associated with Campilobacter Jejuni infection. International Journal of Clinical Case Reports and Reviews. 10(3); DOI: 10.31579/2690-4861/184

Copyright: @ 2022 Kafrouni Hazem, This is an open access article distributed under the Creative Commons Attributiosn License, which permits unrestricted use, distribution, and reproduction in any medium, provided the original work is properly cited.

\begin{abstract}
The pharyngeal-cervical-brachial variant of Guillain-Barré syndrome is a rare but well documented in the adult population, characterized by rapidly progressive weakness of oropharyngeal and cervicobrachial areas with areflexia in the upper limbs. We describe a 19-year-old young man with predominantly pharyngeal-cervical-brachial weakness and pronounced bulbar symptoms. Atypical Guillain-Barré variants can be challenging in early disease stage and should be considered in the differential diagnosis of a young adult presenting with bulbar palsy.

Categories: Internal Medicine, Neurology

Keywords: guillain-barre syndrome (gbs); bilateral glossopharyngeal paralysis; pharyngeal-cervical-brachial variant; campylobacter jejuni
\end{abstract}

\section{Introduction}

Guillain-Barré syndrome (GBS) is a neurologic disease characterized by ascending paralysis, variable degrees of weakness, sensory abnormalities, and autonomic dysfunction. Its etiology is not entirely clarified, though there is evidence to support the hypothesis of a postinfectious autoimmune disorder. Almost 50 to 75 percent of the cases are preceded by an infection, whereby Campylobacter jejuni is the most common identified infectious trigger. Cytomegalovirus, Epstein-Barr virus, Mycoplasma pneumonia and human immunodeficiency virus are also described to be related to this disease. Occasionally there have been reported cases that happen after vaccination of different types [1,2].

The incidence in high-income countries is estimated to be $0.4-4.0$ cases per 100,000 population and is slightly more common in males than females. It can occur at any age, but it becomes more frequent with advancing age $[3,4]$.

GBS is clinically characterized by an acute onset of generalized and symmetrical muscle weakness and areflexia from peripheral nerve involvement. However, other patterns are recognized where there is an unusual distribution of muscle involvement: external ocular muscle involvement with ataxia in Miller Fisher syndrome (MFS), and oropharyngeal, neck and upper limb muscle involvement in pharyngealcervical-brachial (PCB) variant form, which incidence is relatively low $[5,6]$.

\section{Case Presentation}

We present a case of a young man, 19 years old, a soccer player, with asthma, medicated with a rescue inhaler, without other relevant history or family history, was admitted to the Emergency Department due to dysphagia to solids and progressive weakness of the upper limbs with about $24 \mathrm{~h}$ of evolution. He reported acute gastroenteritis about one week before.

The patient denied tobacco, alcohol or illicit drugs consumption, recent travel, recent exposure to unusual plants or animals or other allergens.

On examination, he was alert, afebrile, had no meningeal irritation signs. Syntax and comprehension of language were preserved, although he had some difficulty in articulating the speech. Pupils were isochoric and photoreactive with preserved direct and consensual reflections. Oculomotricity was preserved. He had no nystagmus, blepharoptosis or facial asymmetries. The soft palate could not be adequately raised on phonation and showed some limitation of the lingual protrusion.

On the Medical Research Council (MRC) scale, strength was grade 4 in deltoids, 4 in the right forearm, 3 in the left forearm, and 5 in the lower limbs. Tendon reflexes in the arms were absent, but patella reflexes and Achilles reflexes were normal. Sensory examination was unremarkable.

The computerized angiotomography of supra-aortic vessels and brain showed no alterations. Routine laboratory tests and arterial blood gas analysis were within the normal range. A lumbar puncture was performed, whose cerebrospinal fluid contained $4 \mathrm{cell} / \mathrm{mcl}$, with a protein level of $29.3 \mathrm{mg} / \mathrm{dl}$, the glucose level of $70 \mathrm{mg} / \mathrm{dl}$ and the results of meningitis/encephalitis panel were negative. 
On the second day of hospitalization, he was transferred to the Intensive Unit Care because of the rapidly progressive clinical aggravation with development of flaccid tetraparesis with predominance in the upper limbs, dysarthria, severe dysphagia for solids and liquids, and restrictive pulmonary syndrome requiring continuous aspiration of secretions and a nasogastric tube for feeding. It was performed a magnetic resonance imaging of the head and spine that had no changes. By that time, treatment with intravenous immunoglobulin $(0.4 \mathrm{mg} / \mathrm{Kg} /$ day $)$ was started because of the high suspicion of Guillain-Barré Syndrome. All cultural examinations were negative, except for Campylobacter jejuni PCR was positive in the feces. Serotypes of atypical agents and autoimmunity were negative. Also, antiganglioside antibodies were negative.

On the third day, a nerve conduction study was performed and showed severe neurogenic alterations, at the level of all the studied leads, more accentuated at the level of the upper and distal limbs, with low motor amplitude, but normal conduction velocities, of the radial and posterior tibial nerves bilaterally and common peroneal on the left. The remaining conduction studies (including sensitive studies) were within the normal range.

The clinical situation stabilized and began to improve after the third day of treatment. Percutaneous endoscopic gastrostomy was required due to persistent severe dysphagia. Functional respiratory tests with impaired peak cough flow measurements at the beginning of hospitalization started improving progressively. Even though the patient did not need artificial ventilation or oxygen supplementation, he needed assistance in coughing using a Cough-assist.

He was discharged after 26 days and admitted to a Rehabilitation Center for physical and motor rehabilitation and speech therapy.

After one year of follow-up and rehabilitation, the patient recovered muscle strength of the four limbs and had no speech changes. However, dysphagia was only completely resolved after 18 months.

After one year of follow-up and rehabilitation, the pacient recovered muscle strength of the four limbs and had no speech changes, however dysphagia was only completely resolved after 18 months.

\section{Discussion}

The Pharyngeal-Cervical-Brachial (PCB) variant of Guillain-Barré Syndrome (GBS) is a rare form of presentation, characterized by marked progressive weakness of the oropharynx and neck and proximal upper limbs, usually sparing strength and reflexes of the lower limbs. However, it is documented in literature that some cases, similar to our patient, have had later mild lower limb muscle weakness and/or areflexia, which does not exclude the diagnosis when present. Frequently, there is a rapid progressive pharyngeal weakness, leading to the need for intubation and ventilation due to excessive accumulation of secretions $[5,7,8]$.

The diagnosis of this condition is based on cerebrospinal fluid (CSF) analysis and nerve conduction study, after excluding several possible differential diagnosis. It is expected to have a high CSF protein, as fluid albuminocytological dissociation is present in $42.0 \%$ of the cases [9].

Commonly, electro-physiological studies are characterized by the absence of $F$ waves in upper limbs and rarely in the lower limbs as well. Essentially GBS is divided into three different electrophysiological subtypes: acute inflammatory demyelinating polyneuropathy (AIDP), acute motor axonal neuropathy (AMAN), and acute motor sensory axonal neuropathy (AMSAN) $[9,10,11]$.

Many physicians are unfamiliar about pharyngeal-cervical-brachial variant, which can often be misdiagnosed. Given that our patient is relatively younger than the patients from the majority of the cases in literature, it became important to seek for other possible diagnosis for example: brainstem tumor, neuro-Behçet disease, multiple sclerosis, polymyositis, toxic or metabolic neuropathy, acute encephalomyelitis, Lyme disease, and vascular disease involving the brainstem [7].

The presence of autoantibodies against specific neuronal gangliosides also supports the diagnosis, since there is retrospective study where $68 \%$ of patients with PCB tested positive for at least one of the antiganglioside antibodies, especially GT1a IgG antibodies, which are believed to play a pathogenic role in the development of bulbar palsy in PCB, and were found in $51 \%$ of the patients $[5,9]$.

GBS is often preceded by an infectious illness, characteristically upper respiratory and gastrointestinal infections. Several case studies identified Campylobacter jejuni as the microorganism which most frequently triggers this neuropathy. Although, the risk of developing GBS after this infection is rather low, there's evidence that it is stongly associated with axonal GBS and on rare occasions demyelination can occur $[1,9,11]$.

Intravenous immunoglobulin or plasma exchange are the cornerstone of GBS treatment. Impaired bulbar function frequently requires nasogastric feeding and some patients that develope respiratory insufficiency need ventilatory support. Also physical therapy during recovery is crucial to regain strength and movement capacity [12].

Initially, patients progressively worsen for about one or two weeks, reaching a clinical plateau within four weeks. Recovery usually lasts six to 12 months, though it could take as long as three years [12].

\section{Conclusion}

GBS diagnosis can be complex due to its rarity and heterogeneous clinical presentation, which can be misleading initially. The early recognition of this disease is important to establish a correct diagnosis, change the prognosis with an adequate early treatment and avoid excessive and unnecessary investigations.

\section{References}

1. Allos BM. (1997). Association between Campylobacter infection and Guillain-Barré syndrome. J Infect Dis. 176(S2):125-128.

2. Allen A, Antony SJ. (1999). Severe Guillain-Barré syndrome associated with Campylobacter jejuni infection with failure to respond to plasmapheresis and immunoglobulin. J Am Board Fam Pract. 12(3):249-252.

3. Tam CC, O'Brien SJ, Rodrigues LC. (2006). Influenza, Campylobacter and Mycoplasma infections, and hospital admissions for Guillain-Barré syndrome, England. Emerg Infect Dis. 12(12):1880-1887.

4. Rees JH, Soudain SE, Gregson NA, Hughes RA. (1995). Campylobacter jejuni infection and Guillain-Barré syndrome. N Engl J Med. 333(21):1374-1379.

5. Murakami N, Tomita Y, Koga M, Takahashi E, Katada Y, Sakuta R, Nagai T. (2005). An adolescent with pharyngealcervical-brachial variant of Guillain-Barré syndrome after cytomegalovirus infection. Brain Dev. 28(4):269-271.

6. Arai M, Susuki K, Koga M. (2003). Axonal pharyngeal-cervicalbrachial variant of Guillain-Barré syndrome without Anti-GT1a IgG antibody. Muscle Nerve. 28(2):246-250.

7. Gunasekara, S. \& Wickramasinghe, Chandani. (2019). Pharyngeal-Cervical-Brachial (PCB) variant of Guillain Barre Syndrome. Sri Lanka Journal of Medicine.

8. MacLennan SC, Fahey MC, Lawson JA. (2004). Pharyngealcervical-brachial variant Guillain-Barré syndrome in a child. J Child Neurol. 19(8):626-627. 
9. Nagashima T, Koga M, Odaka M, Hirata K, Yuki N. (2007). Continuous spectrum of pharyngeal-cervical-brachial variant of Guillain-Barré syndrome. Arch Neurol. 64(10):1519-1523.

10. Pradhan RR, Yadav SK, Yadav SK Sr. (2020). PharyngealCervical-Brachial variant of Guillian-Barre Syndrome in Children. Cureus. 12(2):e6983.
11. Drenthen J, Yuki N, Meulstee J, Maathuis EM, van Doorn PA, Visser GH, Blok JH, Jacobs BC. (2011). Guillain-Barré syndrome subtypes related to Campylobacter infection. J Neurol Neurosurg Psychiatry. 82(3):300-305.

12. Wakerley BR, Yuki N. (2014). Pharyngeal-cervical-brachial variant of Guillain-Barré syndrome. Journal of Neurology, Neurosurgery \& Psychiatry. 85:339-344.
This work is licensed under Creative

Commons Attribution 4.0 License
Ready to submit your research? Choose Auctores and benefit from:

$>$ fast, convenient online submission

$>$ rigorous peer review by experienced research in your field

$>$ rapid publication on acceptance

$>$ authors retain copyrights

$>$ unique DOI for all articles

$>$ immediate, unrestricted online access

At Auctores, research is always in progress.

Learn more https://auctoresonline.org/journals/international-journal-of-clinicalcase-reports-and-reviews 